\title{
Artificially Generated Training Datasets for Supervised Machine Learning Techniques in Magnetic Resonance Imaging: An Example in Myocardial Segmentation
}

\author{
Christos G Xanthis ${ }^{1}$, Kostas Haris ${ }^{1}$, Dimitrios Filos ${ }^{1}$, Anthony H Aletras ${ }^{1}$ \\ ${ }^{1}$ Laboratory of Computing, Medical Informatics and Biomedical - Imaging Technologies, School of \\ Medicine, Aristotle University of Thessaloniki, Thessaloniki, Greece
}

\begin{abstract}
Machine learning techniques have become increasingly successful over the last few years in medical image analysis and radiology. However, the low availability, relativeness and size of the training data sets required by the associated learning algorithms prevents their further development or delays their application in clinical practice.

This study presented for the first time the development of artificially generated training datasets for supervised learning techniques through the incorporation of a realistic simulation framework in the field of Magnetic Resonance Imaging (MRI). An example in left-ventricle segmentation was utilized and the performance of a fully convolutional network on true cardiac MR data was evaluated.
\end{abstract}

\section{Introduction}

Machine Learning (ML) has matured today to be used for solving problems of practical nature. The increasing computational power provided by the latest evolvements in technology and the increasing availability of digitized information through the internet unfolded the potential of ML in the daily practice. In the field of radiology, ML has proven to be an effective tool to automate the analysis and diagnosis for medical images. Supervised ML techniques have shown significant potential in medical image analysis and can potentially reduce the burden on physicians in the daily practice of radiology. Examples of supervised machine learning in medical image analysis include image segmentation, image registration but also detection and characterization of abnormalities. However, these techniques come with a major drawback, which either prevents their further development or delays their application in clinical practice. This drawback is related to the availability, relativeness and size of the training data sets required by the associated learning algorithms. The research community has long recognized the importance of this topic.

This study aimed to encounter the low-availability, the poor-relativeness and the small-size of the training datasets that prevent the further development or delay the application of supervised ML techniques in radiology. This study proposes for the first time the development of artificially generated training datasets for supervised learning techniques through the incorporation of a realistic simulation framework in Magnetic Resonance Imaging (MRI).

\section{Methods}

The 4D-XCAT anatomical model [1] was utilized as the main computer model whereas the coreMRI simulation platform (www.coremri.org) [2] was utilized for the generation of artificial MR images. The 4D-XCAT model was modified to be incorporated into coreMRI but also to be adapted to MR applications.

91 artificial midventricular, end-diastolic, short-axis, cardiac MR images were generated in coreMRI and the corresponding maps of the myocardium of the leftventricle were extracted from the $4 \mathrm{D}$ XCAT model. The artificial dataset was further augmented by geometric transformations (rotation, translation, scaling, vertical and horizontal flipping). Then, synthetic white noise was added, and Gaussian filtering was applied. The resulting dataset contained 546 images and was used for the training of the fully convolutional network (FCN) proposed by Tran [3] for LV segmentation. The FCN consisted of 15 stacked convolution layers and three layers of overlapping max pooling of stride 2. Each convolution layer was followed by a Rectified Linear Unit and a Mean-Variance normalization operation. The performance of the NN was evaluated on true cardiac MR data (midventricular, enddiastolic, short-axis, cardiac MR images) that were acquired from one healthy volunteer using a similar MR protocol. The number of training epochs used was 20 and 
the evaluation metrics used were accuracy, Dice index and Jaccard index.

\section{Results}

The application of the FCN (trained on the dataset of the simulated MR images) on the cardiac MR data of the healthy volunteer presented an accuracy of 0.96 and a DICE index of 0.83 for the prediction of epicardium and an accuracy of 0.99 and a DICE index of 0.90 for the prediction of endocardium.

Table 1 summarizes these results whereas Figure 1 shows indicative predictions of the LV contours (epicardium and endocardium) for both synthetic and real MRI images. White contours indicate ground truth.

Table 1. Performance of the fully convolutional network on the cardiac MR data of the healthy volunteer.

\begin{tabular}{lccc}
\hline & Accuracy & $\begin{array}{c}\text { Dice } \\
\text { index }\end{array}$ & $\begin{array}{c}\text { Jaccard } \\
\text { index }\end{array}$ \\
\hline Endocardium & 0.99 & 0.90 & 0.83 \\
Epicardium & 0.96 & 0.83 & 0.73 \\
\hline
\end{tabular}

\section{Conclusion}

This study presented an alternative solution to the limitations encountered today in collecting training datasets that are used in supervised learning techniques in the field of medical MR image analysis. Nowadays, the lack of large training sets does not allow for the development of advanced supervised machine learning techniques, whereas the availability of non-representative training sets may delay the application of these techniques in clinical practice.

The proposed solution is performed without the use of a true MRI scanner, without scanning patients and without requiring personnel (such as technologists for running the MR scanner and radiologists for evaluating the MR images). The digitized and highly customizable nature of the anatomical model will allow for the concurrent production of well-annotated data in the form of quantitative maps, tissue delineations or quantitative values of anatomy and physiology.
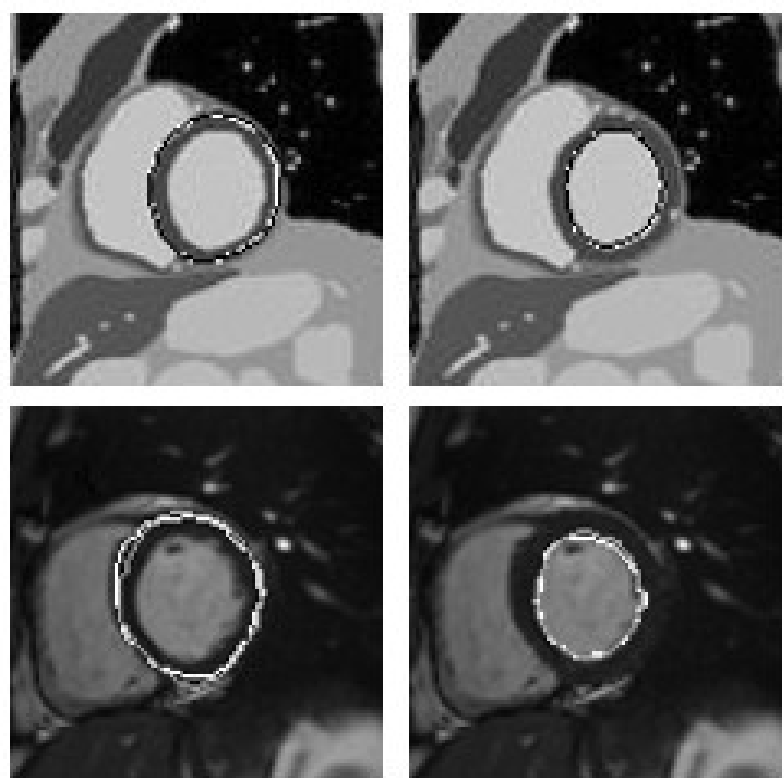

Figure 1. Indicative predictions (dark contours) of the LV contours (epi and endo) for both synthetic (top) and real (bottom) MR images. White contours indicate ground truth.

\section{Acknowledgments}

This project has been co-financed by the Operational Program "Human Resources Development, Education and Lifelong Learning" and is co-financed by the European Union (European Social Fund) and Greek national funds.

\section{References}

[1] W. P. Segars, "4D XCAT phantom for multimodality imaging research," Med Phys., vol. 37, no. 9, pp. 4902-4915, Sep. 2010.

[2] C. G. Xanthis, "coreMRI: A high-performance, publicly available MR simulation platform on the cloud," PLOS $O N E$, vol. 14, no. 5, pp. e0216594, May. 2019.

[3] P. V. Tran, "A fully convolutional neural network for cardiac segmentation in short-axis MRI," arXiv preprint arXiv:1604.00494. Apr. 2017.

Address for correspondence:

Christos G. Xanthis.

Laboratory of Computing, Medical Informatics and Biomedical lmaging Technologies, School of Medicine, Aristotle University of Thessaloniki, PC 54124, Thessaloniki, Greece cxanthis@gmail.com 